\title{
Real Time Attitude Independent GPS Integer Ambiguity Resolution*
}

\author{
E. Glenn Lightsey ${ }^{\dagger}$ and John L. Crassidis ${ }^{\ddagger}$
}

\begin{abstract}
In this paper, a new motion-based approach for Global Positioning System (GPS) integer ambiguity resolution is derived. The approach first represents the GPS sightline vectors in the body frame or the baseline vectors in the reference frame. The solution to this problem is always available as long as at least three non-coplanar baseline or sightline vectors exist. The body-frame sightline or reference-frame baseline observations are the sum of two vectors, one depending on the phase measurements and the other on the unknown integers. The vector containing the integer phases is then converted into an attitude independent observation using scalar checking, which leads to a problem that treats the integers as to-be-determined biases. The bias estimation problem is typically solved by using a batch process. In this paper, simple real time algorithms are developed based on both the extended Kalman filter and Unscented filter. Simulation results indicate that both algorithms provide accurate integer resolution in real time, but the Unscented filter is more robust to large initial condition errors and slow vehicle motions than the extended Kalman filter.
\end{abstract}

\section{Introduction}

When used as a sensor on moving objects, a Global Positioning System (GPS) receiver provides a wealth of information about the vehicle's dynamic state. These data may be used in real time to monitor and potentially control the vehicle's trajectory. The most widely used

*Presented at the AAS John L. Junkins Astrodynamics Symposium, College Station, TX May 23-24, 2003. Paper No. AAS 03-266.

${ }^{\dagger}$ Associate Professor, Center for Space Research, Department of Aerospace Engineering and Engineering Mechanics, University of Texas, Austin, TX 78712-1085. E-mail: lightsey@csr.utexas.edu.

${ }_{\ddagger}^{\ddagger}$ Associate Professor, Department of Mechanical \& Aerospace Engineering, University at Buffalo, State University of New York, Amherst, NY 14260-4400. E-mail: johnc@eng.buffalo.edu. 
dynamic states that are provided by GPS receivers are three dimensional position and velocity along with accurate time measurements. However, GPS receivers have also demonstrated the potential to provide attitude and rotation rate information for the vehicle. The existence of a single low cost sensor that provides both translational and rotational information and is well suited to electronic miniaturization enables numerous real time control applications that were previously considered to be impossible or impractical.

Although vehicle attitude determination using GPS has been demonstrated in some important examples, such as on the International Space Station, ${ }^{1}$ it has not gained widespread acceptance as an alternative to more traditional attitude sensors. The main reason for this reluctance has been a lack of robustness of the attitude solution. Other reasons include a lower attitude accuracy, as compared to other attitude sensors, and a strong dependence of the GPS attitude accuracy on the length of the baselines and their rigidity. The most common method for performing GPS based attitude determination of objects with relatively small dimensions (less than tens of meters) is to measure the difference in the carrier phase signal between multiple antennae which are placed on the object and perform interferometry using the measurements. ${ }^{2}$ The carrier phase signal is used because it has approximately two orders of magnitude greater measurement precision than the GPS code signal. If the separation vector between each of the antennae is known in the object's body frame, then the attitude of the object may be determined with respect to the external reference frame using the differential carrier phase measurements. These operations are reviewed in more detail in the GPS Sensor Model section.

The separation distance between the antennae is almost always greater than one GPS L1 carrier phase wavelength of 19.04 centimeters. Since each antenna measures just the fractional portion of the carrier phase angle, the integer number of wavelengths in the measurement between the two antennae must be determined to convert the differential carrier phase measurement into a differential range measurement. This classic problem in GPS attitude determination is known as the carrier phase integer ambiguity problem, and it has 
been much studied in GPS research. ${ }^{3,4}$ Solving the integer ambiguity is known as integer resolution, and it is this critical step that enables attitude determination to be performed. Once the integers are known, new measurements may be easily incorporated by using the estimated attitude to back-solve for the integers from a newly visible GPS satellite. Using systems of this type, attitude solution accuracies of better than 1 degree are possible with antenna separation distances of only a few meters. ${ }^{2}$

The trouble is that integer resolution is a computationally intense and error prone process, especially in the presence of noisy and/or weak signal measurement environments. Although many algorithms may work relatively well during strong signal and low noise conditions, many of these algorithms break down when the measurement conditions degrade. In these cases, the integer resolution process may go through long periods without a solution or may even report an incorrect solution. The problem is compounded by the fact that it is precisely in these poor measurement environments that the integer resolution algorithm will be called more frequently, due to a lack of available measurements and the presence of cycle slips. This has caused the GPS based attitude determination sensor to display a lack of robustness in some cases. Figure 1 demonstrates this robustness issue using on-orbit data collected in 1996. ${ }^{5}$ Although there are many correct attitude solutions during the primary data collection from $t=75$ to 275 minutes, there are also data dropouts of several minutes, lasting more than 10 minutes in some cases. There is also an incorrect solution reported at approximately $t=175$ minutes.

The problem is made more challenging by the real time nature of the sensor requirements. There are examples of carrier phase integer resolution methods which have been successfully applied to position and attitude determination applications. ${ }^{6}$ However, integer resolution integrity is still an important consideration for real time control applications. Data in the future are not available, and data dropouts and incorrect solutions may produce unacceptable control.

The objective of this paper is to present and compare two sequential integer resolution 
algorithms that are suitable for real time attitude determination applications. The algorithms are attitude independent in the sense that they do not require any prior knowledge of the vehicle attitude to work. The first algorithm uses an extended Kalman filter (EKF) approach that is developed with commonly employed estimation techniques. The second algorithm uses an Unscented filter (UF) approach which offers very good results for robust integer resolution in poor measurement conditions.

\section{Prior Art}

Although many different algorithms have been proposed for integer resolution, most may generally be categorized as one of two types. The first type is known as the search method. Algorithms using the search method attempt to resolve the integers by considering the full set of possible values and picking the best solution for the current measurement sample. ${ }^{7,8}$ A simple implementation of the search method would calculate the solution residual for every possible integer and report the integer set with the minimum overall solution residual. The main appeal of search method algorithms is that they can be very fast under good measurement conditions, often yielding a solution in just a few samples.

Some of the problems with search methods are that they are computationally intense and prone to report no solution or an erroneous solution under poor measurement conditions. The reason that they are computationally intense is that the full set of possible integers can be extremely large even for modestly sized objects of a few meters. It is very difficult to consider all of the possible solutions in a real time application without introducing significant computational latency. However, many search method algorithms have developed innovative techniques to discard families of solutions and thus reduce the computation load. ${ }^{9}$ In some cases, the range of possible attitude solutions is restricted to further reduce the search space. This can help if there is a priori information about the attitude of the vehicle, but it limits the range of operation.

A more fundamental limitation of the search method algorithms is their performance under poor measurement conditions. When measurement error is large, due to multipath 
for example, impostor solutions may masquerade as the correct solution by having a lower solution residual. Since multipath error is geometry dependent, these erroneous solutions will persist until the geometric conditions change enough to allow the correct solution to be revealed. Therefore, a search method algorithm may possibly report the erroneous solution as the correct solution. Additional robustness may be brought into the algorithm by waiting a predetermined amount of time to ensure that the candidate solution is not erroneous, but in this case, the main advantage of the search method has been lost (its quick resolution). Another mechanism for robustness is to require that the solution residual of the candidate solution be much lower than the next best solution, since it is unlikely that an erroneous solution would have such a large separation over the next best solution. In poor measurement conditions, however, this may lead to no solution at all, because the correct solution may never experience such a large separation in residual over the next best solution.

The other type of integer resolution algorithm is known as the motion method. Motion methods rely on the geometry between the antenna array and the GPS satellites to change while the integers remain constant. Therefore, the biases in the measurements may be attributed to the integers and the problem of integer resolution reduces to one of bias determination. The main appeal of the motion method is that the possibility of obtaining an incorrect solution becomes very small as the range of geometric motion becomes large.

There are some problems with motion methods as well. The first is that the geometric motion between the array and the GPS satellites may be very slow in some cases. This reduces the observability of the problem and requires the user to wait longer to obtain a solution. This delay may not be acceptable in a real time control application. If it is not possible to somehow increase the amount of geometric motion (by performing a vehicle rotation, for example), then the algorithm must be designed to work in marginally observable conditions.

If the motion method algorithm is a batch algorithm, then all the measurements must be retained and a single batch solution is sought over the entire data collection. While 
techniques that use this approach have been implemented for attitude determination, ${ }^{10}$ one problem that frequently occurs is the inversion of a very large matrix to obtain a solution. Therefore, it is highly desirable to obtain a sequential formulation of the motion method, if possible, instead of a batch solution.

An attitude independent approach using motion methods is shown in Ref. [11]. This approach represents the GPS sightline vectors in the body frame as the sum of two vectors, one depending on the phase measurements and the other on the unknown integers. The vector containing the integer phases is found using a procedure developed to solve for magnetometer biases ${ }^{12}$ which leads to a linear least squares solution that can also be implemented in real time. In this paper, two new sequential attitude independent integer resolution algorithms are developed using motion methods. These algorithms are also suitable for implementation in real time attitude determination applications. The first algorithm, using an extended Kalman filter approach, is simpler to implement but it does not perform as well under poor measurement conditions. The second algorithm, using an Unscented filter approach, is designed specifically for poor measurement environments and low observability conditions. The algorithms are compared to demonstrate the performance differences.

\section{GPS Sensor Model}

In this section, a brief background of the GPS phase difference measurement is shown. The main measurement used for GPS attitude determination is the phase difference of the signal received from two antennae separated by a baseline. The essential geometry of the wavefront angle and wavelength, which is used to develop a phase difference, is illustrated in Figure 2. The phase difference measurement is obtained by

$$
b_{l} \cos \theta=\lambda(\Delta \phi-n)
$$

where $b_{l}$ is the baseline length (in $\mathrm{cm}$ ), $\theta$ is the angle between the baseline and the line-ofsight to the GPS spacecraft, $n$ is the number of integer wavelengths between two antennae, 
$\Delta \phi$ is the phase difference (in cycles), and $\lambda$ is the wavelength (in cm) of the GPS signal. The two GPS frequency carriers are L1 at $1575.42 \mathrm{MHz}$ and L2 at 1227.6 MHz. As of this writing, non-military applications generally use the L1 frequency, which corresponds to a wavelength of $\lambda=19.04 \mathrm{~cm}$. The measurement model can be expressed by

$$
\Delta \tilde{\phi}=\mathbf{b}^{T} A \mathbf{s}+n+w
$$

where $\Delta \tilde{\phi}$ denotes the single-difference phase measurement, $\mathbf{s} \in \Re^{3}$ is the normalized sightline vector to the GPS spacecraft in a reference frame, typically Earth-Centered-Earth-Fixed (ECEF), ${ }^{13} \mathbf{b} \in \Re^{3}$ is the baseline vector (in wavelengths), which is the relative position vector from one antenna to another, $A \in \Re^{3 \times 3}$ is the (proper and orthogonal) attitude matrix that maps the reference frame to the body frame, and $w$ represents a zero-mean Gaussian measurement error with standard deviation $\varpi$, which is $(0.5 \mathrm{~cm}) / \lambda=0.026$ wavelengths for typical phase noise. ${ }^{2}$ Note that other error sources, such as line biases, may exist in the singledifference phase measurement. Line biases can be eliminated by using double-differenced phase measurements, but the effects of noise are increased. The algorithms presented here are valid for both single and double differenced measurements. At each epoch it is assumed that $M$ baselines and $N$ sightlines exist. The minimum number of baselines and sightlines required to determine the attitude within an ambiguity (arising from an intersection of two cones) is two baselines and two sightlines. ${ }^{14}$ This ambiguity can be easily resolved from the geometry of the sensor array to the GPS satellites though. A mathematically unique attitude solution exists if any additional number of baselines or sightlines are available at a given epoch.

\section{Attitude Independent Observation}

In this section, the attitude independent observation derived from GPS phase difference measurements is reviewed (see Ref. [11] for more details). The case of processing multiple baselines and one sightline at a time to resolve the integer ambiguities (solving for $M$ integers 
for each sightline) is considered first. Other sightlines can be processed in parallel. When at least two sightlines have been processed with multiple baselines, then an attitude can be determined. The derivation of the attitude independent observation begins by representing the available sightline vector in the body frame, $A \mathbf{s}$, as the sum of two components. The first component $\overline{\mathbf{s}}$ is a function of the measured fractional phase measurements, and the second c depends on the unknown integer phase differences: ${ }^{11}$

$$
\begin{gathered}
A \mathbf{s}=\overline{\mathbf{s}}-\mathbf{c} \\
\overline{\mathbf{s}}=B^{-1}\left[\sum_{i=1}^{M} \varpi_{i}^{-2} \Delta \tilde{\phi}_{i} \mathbf{b}_{i}\right] \\
\mathbf{c}=B^{-1}\left[\sum_{i=1}^{M} \varpi_{i}^{-2} n_{i} \mathbf{b}_{i}\right] \\
B=\sum_{i=1}^{M} \varpi_{i}^{-2} \mathbf{b}_{i} \mathbf{b}_{i}^{T}
\end{gathered}
$$

where the subscript $i$ denotes the $i^{\text {th }}$ baseline. Since the measurements are not perfect, Eq. (3a) is replaced by the measurement model

$$
\overline{\mathbf{s}}=A \mathbf{s}+\mathbf{c}+\boldsymbol{\epsilon}
$$

where $\mathbf{c}$ is a constant bias since the baselines are assumed constant, and $\boldsymbol{\epsilon}$ is a zero-mean Gaussian process with covariance $R=B^{-1}$. The three-baseline case will be considered, which is the most common in practice. If more baselines are available, then a three-baseline subset can always be chosen. After the integer phases have been determined using this subset, a refined attitude estimate can be computed using all baselines (i.e., three baselines are sufficient to determine an attitude, which may then be used to resolve the integers corresponding to the other baselines).

To eliminate the dependence on the attitude, the orthogonality of $A$ and Eq. (4) are used 
to give

$$
\begin{aligned}
\|\mathbf{s}\|^{2} & =\|A \mathbf{s}\|^{2}=\|\overline{\mathbf{s}}-\mathbf{c}-\boldsymbol{\epsilon}\|^{2} \\
& =\|\overline{\mathbf{s}}\|^{2}-2 \mathbf{s}^{T} \mathbf{c}+\|\mathbf{c}\|^{2}-2(\overline{\mathbf{s}}-\mathbf{c})^{T} \boldsymbol{\epsilon}+\|\boldsymbol{\epsilon}\|^{2}
\end{aligned}
$$

Next, following Alonso and Shuster ${ }^{12}$ the following effective (scalar) measurement and noise are defined:

$$
\begin{gathered}
z \equiv\|\overline{\mathbf{s}}\|^{2}-\|\mathbf{s}\|^{2} \\
v \equiv 2(\overline{\mathbf{s}}-\mathbf{c})^{T} \boldsymbol{\epsilon}-\|\boldsymbol{\epsilon}\|^{2}
\end{gathered}
$$

Then the effective measurement model is

$$
z=2 \overline{\mathbf{s}}^{T} \mathbf{c}-\|\mathbf{c}\|^{2}+v
$$

where $v$ is approximately Gaussian for small $\boldsymbol{\epsilon}$ with mean and variance given by, respectively,

$$
\mu \equiv E\{v\}=-\operatorname{Tr}(R)
$$

and

$$
\sigma^{2} \equiv E\left\{v^{2}\right\}-\mu^{2}=4(\overline{\mathbf{s}}-\mathbf{c})^{T} R(\overline{\mathbf{s}}-\mathbf{c})+2 \operatorname{Tr}\left(R^{2}\right)
$$

Note that the variance in Eq. (9) is a function of the unknown bias vector c. Equation (6a) is used to compute the actual effective measurement (determined from the known quantities in Eqs. (3b), (3d) and the GPS sightline vector), while Eq. (7) represents the effective measurement model as a function of the unknown bias vector c. Equations (6)-(9) define an attitude independent set of equations because they do not contain the attitude matrix $A$. Reference [11] shows a statistically correct centered estimate algorithm ${ }^{12}$ to determine the bias vector $\mathbf{c}$ (and thus the integers) based on linear least squares, and also shows a linear 
sequential algorithm to determine the bias vector in real time.

If coplanar baselines exist, then another approach using multiple sightlines and one baseline can be used to determine an attitude independent effective measurement. This approach converts the baseline vector into the reference frame:

$$
\begin{gathered}
A^{T} \mathbf{b}=\overline{\mathbf{b}}-\mathbf{c} \\
\overline{\mathbf{b}}=S^{-1}\left[\sum_{j=1}^{N} \varpi_{j}^{-2} \Delta \tilde{\phi}_{j} \mathbf{s}_{j}\right] \\
\mathbf{c}=S^{-1}\left[\sum_{j=1}^{N} \varpi_{j}^{-2} n_{j} \mathbf{s}_{j}\right] \\
S=\sum_{j=1}^{N} \varpi_{j}^{-2} \mathbf{s}_{j} \mathbf{s}_{j}^{T}
\end{gathered}
$$

where the subscript $j$ denotes the $j^{\text {th }}$ sightline. The new effective measurement and noise now follow

$$
\begin{gathered}
z \equiv\|\overline{\mathbf{b}}\|^{2}-\|\mathbf{b}\|^{2} \\
v \equiv 2(\overline{\mathbf{b}}-\mathbf{c})^{T} \boldsymbol{\epsilon}-\|\boldsymbol{\epsilon}\|^{2}
\end{gathered}
$$

where the covariance of $\boldsymbol{\epsilon}$ is now given by $R=S^{-1}$. The new effective measurement model is

$$
z=2 \overline{\mathbf{b}}^{T} \mathbf{c}-\|\mathbf{c}\|^{2}+v
$$

where $v$ is approximately Gaussian for small $\boldsymbol{\epsilon}$ with mean and variance given by, respectively,

$$
\mu \equiv E\{v\}=-\operatorname{Tr}(R)
$$

and

$$
\sigma^{2} \equiv E\left\{v^{2}\right\}-\mu^{2}=4(\overline{\mathbf{b}}-\mathbf{c})^{T} R(\overline{\mathbf{b}}-\mathbf{c})+2 \operatorname{Tr}\left(R^{2}\right)
$$


This approach has the advantage of determining the integers using one baseline at a time (thus the non-coplanar baseline requirement of the previous approach is not required); however, at least three GPS sightlines must be available until the integer solutions have converged. Also, note that the vector $\mathbf{c}$ is no longer constant, but unlike the approach shown in Ref. [11], this poses no difficulties in the EKF and UF formulations.

\section{Kalman Filter Formulation}

In this section, an extended Kalman filter (EKF) is derived to determine the integers in real time. A review of the EKF equations can be found in Ref. [15]. The filter output and state dynamics for the GPS integer ambiguity problem are straightforward. The output is given by the effective measurement generated using Eq. (6a) and the state vector is defined as a vector of the integers, so that $\mathbf{x} \equiv\left[\begin{array}{lll}n_{1} & n_{2} & n_{3}\end{array}\right]^{T}$. Since this vector is known to be constant, then the filter dynamics are given by

$$
\dot{\hat{\mathbf{x}}}=\mathbf{0}
$$

where $\hat{\mathbf{x}}$ denotes an estimate of $\mathbf{x}$. Since the goal is parameter estimation, then the process noise covariance is simply zero. Therefore, the integers are strictly determined by the discrete-time Kalman update equations, given by

$$
\begin{gathered}
\hat{\mathbf{x}}_{k+1}=\hat{\mathbf{x}}_{k}+K_{k}\left[z_{k+1}-h_{k+1}\left(\hat{\mathbf{x}}_{k}\right)\right] \\
P_{k+1}=\left[I_{3 \times 3}-K_{k} H_{k+1}\left(\hat{\mathbf{x}}_{k}\right)\right] P_{k} \\
K_{k}=P_{k} H_{k+1}^{T}\left(\hat{\mathbf{x}}_{k}\right)\left[H_{k+1}\left(\hat{\mathbf{x}}_{k}\right) P_{k} H_{k+1}^{T}\left(\hat{\mathbf{x}}_{k}\right)+\sigma_{k+1}^{2}\left(\hat{\mathbf{x}}_{k}\right)\right]^{-1}
\end{gathered}
$$


where $\hat{\mathbf{x}}_{k}=\left[\begin{array}{lll}\hat{x}_{1_{k}} & \hat{x}_{2_{k}} & \hat{x}_{3_{k}}\end{array}\right]^{T}$, which represents the estimates for $n_{1}, n_{2}$ and $n_{3}, P_{k}$ is the state error-covariance, and

$$
\begin{gathered}
h_{k+1}\left(\hat{\mathbf{x}}_{k}\right)=2 \overline{\mathbf{s}}_{k+1}^{T} \mathbf{c}\left(\hat{\mathbf{x}}_{k}\right)-\left\|\mathbf{c}\left(\hat{\mathbf{x}}_{k}\right)\right\|^{2} \\
\mathbf{c}\left(\hat{\mathbf{x}}_{k}\right)=B^{-1}\left(\varpi_{1}^{-2} \hat{x}_{1_{k}} \mathbf{b}_{1}+\varpi_{2}^{-2} \hat{x}_{2_{k}} \mathbf{b}_{2}+\varpi_{3}^{-2} \hat{x}_{3_{k}} \mathbf{b}_{3}\right) \\
\sigma_{k+1}^{2}\left(\hat{\mathbf{x}}_{k}\right)=4\left[\overline{\mathbf{s}}_{k+1}-\mathbf{c}\left(\hat{\mathbf{x}}_{k}\right)\right]^{T} B^{-1}\left[\overline{\mathbf{s}}_{k+1}-\mathbf{c}\left(\hat{\mathbf{x}}_{k}\right)\right]+2 \operatorname{Tr}\left(B^{-2}\right)
\end{gathered}
$$

The $1 \times 3$ matrix $H_{k+1}\left(\hat{\mathbf{x}}_{k}\right)$ is derived by taking the partial of Eq. (7) with respect to $\mathbf{x}$, which gives

$$
H_{k+1}\left(\hat{\mathbf{x}}_{k}\right)=2\left[\overline{\mathbf{s}}_{k+1}-\mathbf{c}\left(\hat{\mathbf{x}}_{k}\right)\right] B^{-1}\left[\begin{array}{lll}
\varpi_{1}^{-2} \mathbf{b}_{1} & \varpi_{2}^{-2} \mathbf{b}_{2} & \varpi_{3}^{-2} \mathbf{b}_{3}
\end{array}\right]
$$

The notations $h_{k+1}\left(\hat{\mathbf{x}}_{k}\right), H_{k+1}\left(\hat{\mathbf{x}}_{k}\right)$ and $\sigma_{k+1}^{2}\left(\hat{\mathbf{x}}_{k}\right)$ denote an evaluation at the $k+1$ time-step measurement using $\overline{\mathbf{s}}_{k+1}$ and at the $k$ time-step estimate using $\hat{\mathbf{x}}_{k}$. As previously stated the variance of the effective measurement error contains the unknown vector $\mathbf{c}$. In order to implement this condition in the EKF formulation it is assumed that the measurement variance is given by $\sigma_{k+1}^{2}\left(\hat{\mathbf{x}}_{k}\right)$, where the estimated bias vector is used at each update. Although this approach is not truly "optimal" in the strictness sense, it is the simplest approach to implement in practice. Furthermore, simulation studies indicate that the EKF is not sensitive to errors in the measurement variance when using the current estimate.

The implementation of the EKF for GPS integer ambiguity resolution proceeds as follows. First, given three phase difference measurements associated with three baseline vectors and a sightline vector, compute an effective measurement at time $t_{0}$ using Eqs. (3b), (3d) and (6a). Initialize the filter with some initial covariance estimate $P_{0}$ and state estimate $\mathbf{x}_{0}$. Then, use the current estimate to update the state and covariance at each measurement time by the update equations shown in Eq. (16). At each time step a new effective measurement is computed and the filter is executed in real time until convergence is achieved. We should note that the estimates given by the filter are not explicitly integers. The integer ambiguities can be resolved by rounding these estimates to the nearest integers. The diagonal elements of the 
covariance matrix, denoted by $P_{i i}(i=1,2,3)$, can be used to develop a stopping criterion. In particular, the procedure of rounding an estimate to the nearest integer can be shown to have a 0.0013 probability of selecting the wrong integer when $3 \sqrt{P_{i i}}$ is less than one-half. ${ }^{11}$ This approach also has the advantage of checking "how close" the estimated values are to their closest integer values. Multiple sightline vectors can be processed in parallel. When the filter has converged for two or more sightlines, then an attitude can be computed, which can be used to instantaneously resolve the integer ambiguities associated with other sightlines.

If coplanar baselines exist, then the baselines can be converted into the reference frame using Eqs. (10b) and (10d), which requires that three non-coplanar sightlines exist throughout the entire time span. This approach uses Eq. (11a) to compute the effective measurement. The quantities in the EKF of Eq. (16) now are given by

$$
\begin{gathered}
h_{k+1}\left(\hat{\mathbf{x}}_{k}\right)=2 \overline{\mathbf{b}}_{k+1}^{T} \mathbf{c}_{k+1}\left(\hat{\mathbf{x}}_{k}\right)-\left\|\mathbf{c}_{k+1}\left(\hat{\mathbf{x}}_{k}\right)\right\|^{2} \\
\mathbf{c}_{k+1}\left(\hat{\mathbf{x}}_{k}\right)=S_{k+1}^{-1}\left(\varpi_{1}^{-2} \hat{x}_{1_{k}} \mathbf{s}_{1_{k+1}}+\varpi_{2}^{-2} \hat{x}_{2_{k}} \mathbf{s}_{2_{k+1}}+\varpi_{3}^{-2} \hat{x}_{3_{k}} \mathbf{s}_{3_{k+1}}\right) \\
\sigma_{k+1}^{2}\left(\hat{\mathbf{x}}_{k}\right)=4\left[\overline{\mathbf{b}}_{k+1}-\mathbf{c}_{k+1}\left(\hat{\mathbf{x}}_{k}\right)\right]^{T} S_{k+1}^{-1}\left[\overline{\mathbf{b}}_{k+1}-\mathbf{c}_{k+1}\left(\hat{\mathbf{x}}_{k}\right)\right]+2 \operatorname{Tr}\left(S_{k+1}^{-2}\right)
\end{gathered}
$$

The $1 \times 3$ matrix $H_{k+1}\left(\hat{\mathbf{x}}_{k}\right)$ is derived by taking the partial of Eq. (12) with respect to $\mathbf{x}$, which gives

$$
H_{k+1}\left(\hat{\mathbf{x}}_{k}\right)=2\left[\overline{\mathbf{b}}_{k+1}-\mathbf{c}_{k+1}\left(\hat{\mathbf{x}}_{k}\right)\right] S_{k+1}^{-1}\left[\begin{array}{lll}
\varpi_{1}^{-2} \mathbf{s}_{1_{k+1}} & \varpi_{2}^{-2} \mathbf{s}_{2_{k+1}} & \varpi_{3}^{-2} \mathbf{s}_{3_{k+1}}
\end{array}\right]
$$

Note that the matrix $S$ is time-varying. Therefore, a $3 \times 3$ matrix inverse is required at each measurement update to use this approach.

The EKF formulation presented here is different than the sequential least squares process of Ref. [11], where a centering algorithm is first used to remove the nonlinearities in the derived cost function, which then yields a simple linear least squares solution. This, in turn, can easily be executed in real time using a sequential process. The linear sequential process works well when sufficient vehicle or GPS satellite motion is present; however, as shown by 
simulation results, the sequential least squares process may produce erroneous results when the motion is marginally observable in the presence of measurement noise. Furthermore, the approach of Ref. [11] requires that the vector $\mathbf{c}$ be constant, which is only true when using the sightline vector in the body frame to create the effective measurement. The EKF is used to estimate the integers directly, so c need not be constant. This clearly has advantages when three coplanar baselines exist.

\section{Unscented Filter Formulation}

In this section a new approach, developed by Julier, Uhlmann and Durrant-Whyte, ${ }^{16}$ is discussed as an alternative to the EKF. This approach, which they called the Unscented filter (UF), works on the premise that with a fixed number of parameters it should be easier to approximate a Gaussian distribution than to approximate an arbitrary nonlinear function. The Unscented filter uses a different propagation than the form given by the standard extended Kalman filter. Given an $n \times n$ covariance matrix $P$, a set of order $n$ points can be generated from the columns (or rows) of the matrices $\pm \sqrt{n P}$. The set of points is zero-mean, but if the distribution has mean $\boldsymbol{\mu}$, then simply adding $\boldsymbol{\mu}$ to each of the points yields a symmetric set of $2 n$ points having the desired mean and covariance. Due to the symmetric nature of this set, its odd central moments are zero, so its first three moments are the same as the original Gaussian distribution (see Ref. [17] for more details).

The implementation of the UF for real-time GPS integer ambiguity resolution is straightforward. First, the following set of sigma points are computed from $P_{k}$ :

$$
\begin{gathered}
\boldsymbol{\sigma}_{k} \leftarrow 2 n \text { columns from } \pm \gamma \sqrt{P_{k}} \\
\chi_{k}(0)=\hat{\mathbf{x}}_{k} \\
\chi_{k}(i)=\boldsymbol{\sigma}_{k}(i)+\hat{\mathbf{x}}_{k}, \quad i=1,2, \ldots, 2 n
\end{gathered}
$$

The parameter $\gamma$ is given by $\gamma=\sqrt{n+\lambda}$, where the composite scaling parameter, $\lambda$, is given by $\lambda=\alpha^{2}(n+\kappa)-n$. The constant $\alpha$ determines the spread of the sigma points and is 
usually set to a small positive value (e.g. $\left.1 \times 10^{-4} \leq \alpha \leq 1\right) .{ }^{17}$ Also, the parameter $\kappa$ is usually given by $\kappa=3-n$. Efficient methods to compute the matrix square root can be found by using the Cholesky decomposition. ${ }^{18}$ The following weights are now defined:

$$
\begin{gathered}
W_{0}^{\text {mean }}=\frac{\lambda}{n+\lambda} \\
W_{0}^{\text {cov }}=\frac{\lambda}{n+\lambda}+\left(1-\alpha^{2}+\beta\right) \\
W_{i}^{\text {mean }}=W_{i}^{\text {cov }}=\frac{1}{2(n+\lambda)}, \quad i=1,2, \ldots, 2 n
\end{gathered}
$$

where $\beta$ is used to incorporate prior knowledge of the distribution (for Gaussian distributions $\beta=2$ is optimal).

Since the state model estimate is given by $\dot{\hat{\mathbf{x}}}=\mathbf{0}$, then the propagated values for the state and covariance are given by their respective updated values, which significantly reduces the computational requirements in the UF. Hence, the only essential difference between the EKF and UF formulations is in the computation of the innovations covariance, where the EKF uses a first-order expansion to compute this quantity, while the UF uses a nonlinear transformation to compute this quantity. For the GPS integer ambiguity algorithm using the UF, the state estimate is calculated by

$$
\hat{\mathbf{x}}_{k+1}=\hat{\mathbf{x}}_{k}+K_{k}\left[z_{k+1}-\hat{z}_{k+1}\left(\chi_{k}\right)\right]
$$

where $\hat{z}_{k+1}\left(\chi_{k}\right)$ is the mean observation, given by

$$
\hat{z}_{k+1}\left(\boldsymbol{\chi}_{k}\right)=\sum_{i=0}^{2 n} W_{i}^{\text {mean }} h_{k+1}\left[\chi_{k}(i)\right]
$$

where $h_{k+1}\left[\chi_{k}(i)\right]$ is defined by Eq. (17a) or Eq. (19a), depending on the approach taken. The gain $K_{k}$ is computed by

$$
K_{k}=P_{k}^{x z}\left[P_{k}^{z z}+\sigma_{k+1}^{2}\left(\hat{\mathbf{x}}_{k}\right)\right]^{-1}
$$


where $P_{k}^{x z}$ is the cross-correlation matrix between $\hat{\mathbf{x}}_{k}$ and $\hat{z}_{k+1}\left(\boldsymbol{\chi}_{k}\right)$, given by

$$
P_{k}^{x z}=\sum_{i=0}^{2 n} W_{i}^{\text {cov }}\left\{\boldsymbol{\chi}_{k}^{x}(i)-\hat{\mathbf{x}}_{k}\right\}\left\{h_{k+1}\left[\boldsymbol{\chi}_{k}(i)\right]-\hat{z}_{k+1}\left(\boldsymbol{\chi}_{k}\right)\right\}^{T}
$$

and $P_{k}^{z z}$ is the output covariance, given by

$$
P_{k}^{z z}=\sum_{i=0}^{2 n} W_{i}^{\mathrm{cov}}\left\{h_{k+1}\left[\boldsymbol{\chi}_{k}(i)\right]-\hat{z}_{k+1}\left(\boldsymbol{\chi}_{k}\right)\right\}\left\{h_{k+1}\left[\boldsymbol{\chi}_{k}(i)\right]-\hat{z}_{k+1}\left(\boldsymbol{\chi}_{k}\right)\right\}^{T}
$$

Finally, the propagated covariance is given by

$$
P_{k+1}=P_{k}-K_{k}\left[P_{k}^{z z}+\sigma_{k+1}^{2}\left(\hat{\mathbf{x}}_{k}\right)\right] K_{k}^{T}
$$

New sigma points can now be calculated using $P_{k+1}$ for the sequential UF process.

The implementation of the UF for GPS integer ambiguity resolution proceeds as follows. For brevity only the body-frame (sightline) effective measurement is discussed here. The only essential difference between the EKF and UF formulations is in the computation of the innovations covariance, where the EKF uses a first-order expansion to compute this quantity, while the UF uses a nonlinear transformation to compute this quantity. Another approach for the UF uses the measurement noise model of Eq. (9) with an augmented vector given by the state and $\boldsymbol{\epsilon}$. Therefore, a decomposition of a $6 \times 6$ matrix is now required. In the strictest sense this approach is more optimal than the first approach because the effect of the nonlinear-appearing measurement noise is directly used in the UF. But, the computational requirements are vastly increased due to the decomposition of a higher dimensional augmented matrix. Also, from numerous simulation trials no apparent advantages to using the augmented approach in the UF is seen. More details on this UF formulation for GPS integer ambiguity resolution can be found in Ref. [19]. 


\section{Simulation Results}

In this section, simulation results involving various ground vehicle motions are shown to demonstrate the performance of the new EKF and UF algorithms for GPS integer ambiguity resolution. Figure 3 shows the coordinates systems used to generate simulated data. The Earth-Centered-Earth-Fixed (ECEF) coordinate system has its $z$ axis through the true north pole (i.e., along the Earth's spin axis) and its $x$ axis through the intersection of the prime meridian $\left(0^{\circ}\right.$ longitude) and the equator $\left(0^{\circ}\right.$ latitude). The ECEF $y$ axis completes the righthanded coordinate system. The North-East-Down (NED) coordinate system (also known as the local navigation frame) is defined by fitting a tangent plane onto the surface of the Earth at the particular point of interest. The plane remains fixed about this point and this point becomes the origin of the frame. The NED $x$ axis points to true north, the $y$ axis points east and the $z$ axis points towards the center of the Earth. The body coordinate system is assumed to be rigidly attached to the vehicle undergoing motion. The body $x$ axis points forward, the $z$ axis points down, and the $y$ axis completes the right-handed coordinate system. The heading angle, $\psi$, in Figure 3 is the angle from the NED $x$ axis to the body $x$ axis. The transformation from the ECEF frame to the body frame is given by ${ }^{20}$

$$
\left[\begin{array}{l}
x \\
y \\
z
\end{array}\right]_{\mathrm{BODY}}=\left[A_{\mathrm{NED} 2 \mathrm{BODY}}\right]\left[A_{\mathrm{ECEF} 2 \mathrm{NED}}\right]\left[\begin{array}{l}
x \\
y \\
z
\end{array}\right]_{\mathrm{ECEF}}
$$


with

$$
\begin{aligned}
{\left[A_{\text {NED2BOdy }}\right]=\left[\begin{array}{ccc}
\cos \psi & \sin \psi & 0 \\
-\sin \psi & \cos \psi & 0 \\
0 & 0 & 1
\end{array}\right] } \\
{\left[A_{\mathrm{ECEF} 2 \mathrm{NED}}\right]=\left[\begin{array}{ccc}
-\sin \lambda \cos \phi & -\sin \lambda \sin \phi & \cos \lambda \\
\sin \phi & \cos \phi & 0 \\
-\cos \lambda \cos \phi & -\cos \lambda \sin \phi & -\sin \lambda
\end{array}\right] }
\end{aligned}
$$

where $\lambda$ is the latitude and $\phi$ is the longitude.

The vehicle is assumed to have coordinates of $38^{\circ} \mathrm{N}$ and $77^{\circ} \mathrm{W}$ (i.e., in Washington, DC). The GPS constellation is simulated using GPS week 137 and a time of applicability of 61440.0000 seconds (see Ref. [21] for an explanation of GPS time).* The available GPS satellites are determined using a $15^{\circ}$ cutoff. $^{21}$ A 60 minute simulation is conducted and measurements are sampled at 1 second intervals. Several GPS sightline vectors are available throughout the entire simulation interval. Two vehicle motions are assumed. The first has the vehicle turning at a fairly fast rate of $\dot{\psi}=10 \mathrm{deg} / \mathrm{sec}$, while the second has the vehicle turning at a much slower rate of $\dot{\psi}=1 \mathrm{deg} / \mathrm{sec}$. The GPS attitude sensor frame is assumed to coincide with the body frame. The GPS baseline vectors in wavelengths are assumed to be given by

$$
\mathbf{b}_{1}=\left[\begin{array}{c}
6 \\
0 \\
0
\end{array}\right], \quad \mathbf{b}_{2}=\left[\begin{array}{l}
0 \\
6 \\
0
\end{array}\right], \quad \mathbf{b}_{3}=\left[\begin{array}{c}
0 \\
-2 \\
6
\end{array}\right]
$$

The true integers for all simulations are given by $n_{1}=1, n_{2}=-2$ and $n_{3}=3$. Measurements are generated by adding zero-mean Gaussian white-noise, with a standard deviation of $\varpi=$ 0.026 wavelengths, to the true phase difference observations. Also, multipath errors are introduced using a simple Markov process with time constant of 5 minutes and standard

${ }^{*}$ The U.S. Coast Guard Navigation Center maintains a website that contains GPS almanacs, and as of this writing this website is given by http://www.navcen.uscg.gov/. 
deviation of 0.25 , which is 10 times the amount used in the simulations of Ref. [11].

For the first simulation the fast rate of $\dot{\psi}=10 \mathrm{deg} / \mathrm{sec}$ is used. For this simulation the initial covariance for both the UF and EKF is given by $P_{0}=(16 / 9) I_{3 \times 3}$, which assumes a $3 \sigma$ error-bound of the integers of 4 cycles. The parameters used in the UF are $\alpha=0.1$, $\beta=2, \kappa=3-L$, and $L=3$ when the $3 \times 3$ matrix decomposition approach is used $(L=6$ for the augmented approach). For this simulation run no noticeable differences between the results of the EKF and UF are seen. Also, the two UF approaches (one uses the current state estimate in the measurement-error variance calculation, which requires a $3 \times 3$ matrix decomposition, while the other appends the state vector to include the measurement noise, which requires a $6 \times 6$ matrix decomposition) give identical results. Therefore, only the EKF results are shown. Plots of the integer errors and $3 \sigma$ integer bounds are shown in Figure 4. The top plot of Figure 4(i) shows the estimated solutions minus the true integers, while the bottom plot shows the errors rounded to the nearest integer. The top plot of Figure 4(i) shows that the EKF and UF algorithms can handle large multipath errors well because the errors converge to very small values, which is not the case with the sequential process of Ref. [11]. From Figure 4(ii) the $3 \sigma$ integers bounds fall below $1 / 2$ at around the 30 second point, which coincide with the converged solutions shown by Figure 4(i). Several runs with different random noise seeds have also been executed (i.e., a Monte Carlo type simulation), which all yield the same results shown here. Therefore, both the EKF and UF algorithms work well when the vehicle exhibits moderately fast motions.

For the second simulation the slower rate of $\dot{\psi}=1 \mathrm{deg} / \mathrm{sec}$ is used. For this simulation the initial covariance for both the UF and EKF is given by $P_{0}=4 I_{3 \times 3}$, which assumes a $3 \sigma$ error-bound of the integers of 6 cycles. This essentially assumes that the initial integer estimates are completely unknown, which may range over the entire length of a baseline (e.g., $\left.\left\|\mathbf{b}_{1}\right\|=6\right)$. The parameters for the UF are the same as the ones used in first simulation. For this current simulation run, the results of the EKF solutions and UF solutions are different. The two UF approaches still give identical results, so no apparent advantages of appending 
the state vector to include the nonlinear-appearing measurement noise are seen for this simulation either. Plots of the EKF and UF integer errors and $3 \sigma$ integer bounds are shown in Figure 5. From Figure 5(i), the EKF solutions converge in about 5 minutes, while the UF solutions converge in less time (about 1 minute less). Figure 5(ii) shows the errors rounded to the nearest integer. The integer associated with the third baseline takes the most time to converge because this baseline vector is closest to the axis of the vehicle's rotation. In other cases, using different random noise seeds, the EKF never converges to the correct solution (4 out of 100 Monte Carlo passes did not converge). This is due to the fact that the first-order approximation in the EKF cannot adequately capture the large errors introduced by the initial covariance and the small observability of the system due to the slower rate motion of the vehicle. The sequential least squares process of Ref. [11] does not converge either using the same simulation parameters and data. The UF outperforms the EKF and sequential least squares process in every simulated test case.

The biggest concern with the EKF results is the confidence of the integer solutions dictated by the $3 \sigma$ bounds, shown by the top plot of Figure 5(iii). These bounds indicate that the EKF solutions have converged almost immediately, which is clearly incorrect. Fortunately, the UF $3 \sigma$ bounds, shown by the bottom plot of Figure 5(iii), fall below 1/2 at around the 4 minute point, which coincide with the correctly converged solutions shown by Figure 5(ii). However, the UF algorithm comes with a computational cost. An increased amount of computation is required for the covariance decomposition and multiple output calculations. In this study, the UF algorithm is about 1.5 times slower than the EKF algorithm in execution speed. Still, the performance enhancements of the UF over the EKF outweigh the increased computational costs.

The last simulation involves using three sightlines and one baseline to determine the integers. Equation (11a) is used as the effective measurement for this case. Several sightlines are available over a 10 minute span, and a subset of the three sightlines with the best Geometric Dilution of Precision ${ }^{2}$ is chosen. For this simulation the fast rate of $\dot{\psi}=10$ 
$\mathrm{deg} / \mathrm{sec}$ is used. Also, the initial covariance for the UF is given by $P_{0}=4 I_{3 \times 3}$. Baseline $\mathbf{b}_{1}$ from Eq. (31) is chosen as the baseline for the effective measurement. A plot of the rounded integer errors and $3 \sigma$ bounds for the UF is shown in Figure 6. The UF is able to accurately determine the integers for this case. However, the $3 \sigma$ bounds indicate that convergence takes longer than the results shown in Figure 4, which is most likely due to the geometry of the sightlines leading to a less observable system for this simulation. Still, the reference-frame baseline approach is useful when three coplanar baselines exist because the integers associated with each baseline can be determined independently.

\section{Conclusions}

In this paper, two new real time algorithms based on the extended Kalman filter and Unscented filter were developed for GPS integer ambiguity resolution. The algorithms have several advantages over existing approaches, including: no a priori attitude knowledge is required, the algorithms can easily be modified to work with coplanar baselines, and a suitable integrity check can be used to determine when the estimated values have converged to the correct values. Two different approaches have also been shown in the Unscented filter design. One uses the current state estimate in the measurement-error variance calculation, which requires a $3 \times 3$ matrix decomposition, while the other appends the state vector to include the nonlinear-appearing measurement noise, which requires a $6 \times 6$ matrix decomposition. Simulation results indicated that for this problem, both Unscented filter approaches gave identical results. So appending the state vector is not required, which significantly reduces the computational requirements in the Unscented filter. Simulation test cases also indicated that the performance of the Unscented filter is significantly better than the standard extended Kalman filter for large initialization errors and slow vehicle motions. Hence, the Unscented filter algorithm is recommended for actual implementation. 


\section{References}

${ }^{1}$ GOMEZ, S., "Flying High: GPS on the International Space Station and Crew Return Vehicle," GPS World, Vol. 13, No. 6, June 2002, pp. 12-16.

${ }^{2}$ COHEN, C. E., "Attitude Determination," Global Positioning System: Theory and Applications, edited by B. Parkinson and J. Spilker, Vol. 64 of Progress in Astronautics and Aeronautics, chap. 19, American Institute of Aeronautics and Astronautics, Washington, DC, 1996.

${ }^{3}$ MISRA, P. and ENGE, P., Global Positioning System: Signals, Measurements, and Performance, Ganga-Jamuna Press, Lincoln, MA, 2001.

${ }^{4}$ KAPLAN, E. D., GPS Principles and Applications, Artech House Publishers, Boston, MA, 1996.

${ }^{5}$ CARPENTER, J. R. and HAIN, R. M., "Precise Evaluation of Orbital GPS Attitude Determination on the STS 77 GPS Attitude and Navigation Experiment (GANE)," Proceedings of the Institute of Navigation National Technical Meeting, Los Angeles, CA, January 1997, pp. 387-398.

${ }^{6}$ TEUnissen, P., JONGE, P. D., and TIBERIUS, C., "Performance of the LAMBDA Method for Fast GPS Ambiguity Resolution," Navigation: Journal of the Institute of Navigation, Vol. 44, No. 3, Fall 1997, pp. 373-383.

${ }^{7}$ HATCH, R., "Instantaneous Ambiguity Resolution," KIS Symposium, Banff, Canada, September 1990.

${ }^{8}$ KNIGHT, D., "A New Method of Instantaneous Ambiguity Resolution," Proceedings of the Institute of Navigation GPS-94, Salt Lake City, UT, September 1994.

${ }^{9}$ LIGHTSEY, E. G., CRASSIDIS, J. L., and MARKLEY, F. L., "Fast Integer Am-

biguity Resolution for GPS Attitude Determination," Proceedings of the AIAA Guidance, Navigation, and Control Conference, Portland, OR, August 1999, pp. 403-412. 
${ }^{10}$ LIGHTSEY, E. G., Development and Flight Demonstration of a GPS Receiver for Space, Ph.D. Dissertation, Stanford University, Stanford, CA, 1997.

${ }^{11}$ CRASSIDIS, J. L., MARKLEY, F. L., and LIGHTSEY, E. G., "Global Positioning System Integer Ambiguity Resolution Without Attitude Knowledge," Journal of Guidance, Control, and Dynamics, Vol. 22, No. 2, March-April 1999, pp. 212-218.

${ }^{12}$ ALONSO, R. and SHUSTER, M. D., "TWOSTEP: A Fast Robust Algorithm for Attitude-Independent Magnetometer-Bias Determination," Journal of the Astronautical Sciences, Vol. 50, No. 4, Oct.-Dec. 2002, pp. 433-451.

${ }^{13}$ BATE, R. R., MUELLER, D. D., and WHITE, J. E., Fundamentals of Astrodynamics, Dover Publications, New York, NY, 1971.

${ }^{14}$ CRASSIDIS, J. L. and MARKLEY, F. L., "New Algorithm for Attitude Determination Using Global Positioning System Signals," Journal of Guidance, Control, and Dynamics, Vol. 20, No. 5, Sept.-Oct. 1997, pp. 891-896.

${ }^{15}$ CRASSIDIS, J. L. and JUNKINS, J. L., Optimal Estimation of Dynamic Systems, Chapman \& Hall/CRC, Boca Raton, FL, 2004.

${ }^{16}$ JULIER, S. J., UHLMANN, J. K., and DURRANT-WHYTE, H. F., "A New Method for the Nonlinear Transformation of Means and Covariances in Filters and Estimators," IEEE Transactions on Automatic Control, Vol. AC-45, No. 3, March 2000, pp. 477-482.

${ }^{17}$ WAN, E. and VAN DER MERWE, R., "The Unscented Kalman Filter," Kalman Filtering and Neural Networks, edited by S. HAYKIN, chap. 7, Wiley, 2001.

${ }^{18}$ GOLUB, G. H. and VAN LOAN, C. F., Matrix Computations, The Johns Hopkins University Press, Baltimore, MD, 2nd ed., 1989.

${ }^{19}$ LIGHTSEY, E. G. and CRASSIDIS, J. L., "Real Time Attitude Independent GPS Integer Ambiguity Resolution," John L. Junkins Astrodynamics Symposium, College Station, TX, May 2003, AAS 03-266. 
${ }^{20}$ FARRELL, J. and BARTH, M., The Global Positioning System \& Inertial Navigation, McGraw-Hill, New York, NY, 1998.

${ }^{21}$ HOFMANN-WELLENHOF, B., LICHTENEGGER, H., and COLLINS, J., GPS: Theory and Practice, Springer Wien, New York, NY, 5th ed., 2001. 


\section{List of Figures}

1 On-orbit GPS Carrier Phase Attitude Solutions (Data from GPS Attitude and Navigation Experiment (GANE), May 1996) . . . . . . . . . 26

2 GPS Wavelength and Wavefront Angle . . . . . . . . . . . . . . . 27

3 Earth-Centered-Earth-Fixed, North-East-Down and Body Frames . . . . . . 28

4 Integer Errors and $3 \sigma$ Integer Bounds . . . . . . . . . . . . . . . . . . . 29

$5 \quad$ EKF and UF Integer Errors and $3 \sigma$ Integer Bounds . . . . . . . . . . . . 30

6 Rounded Integer Errors and $3 \sigma$ Integer Bounds for the UF . . . . . . . . . 31 


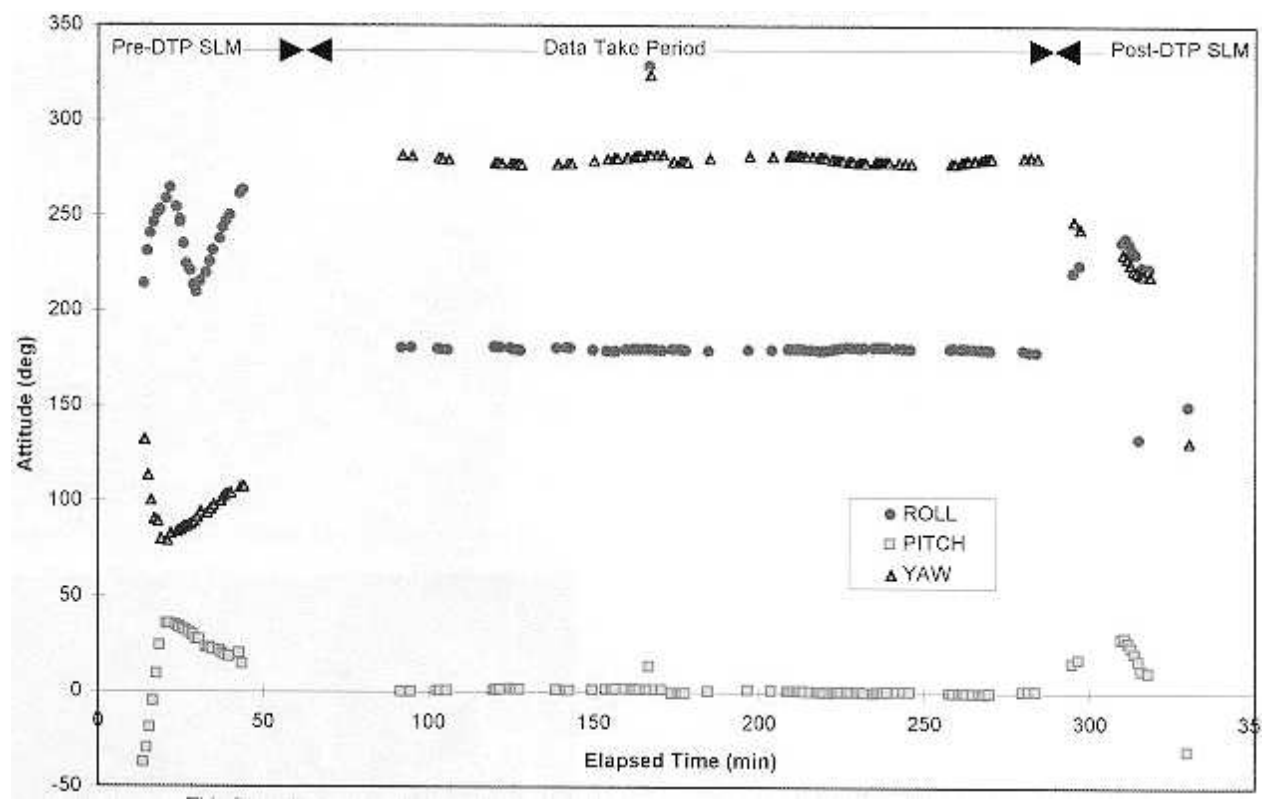

Figure 1: On-orbit GPS Carrier Phase Attitude Solutions (Data from GPS Attitude and Navigation Experiment (GANE), May 1996) 


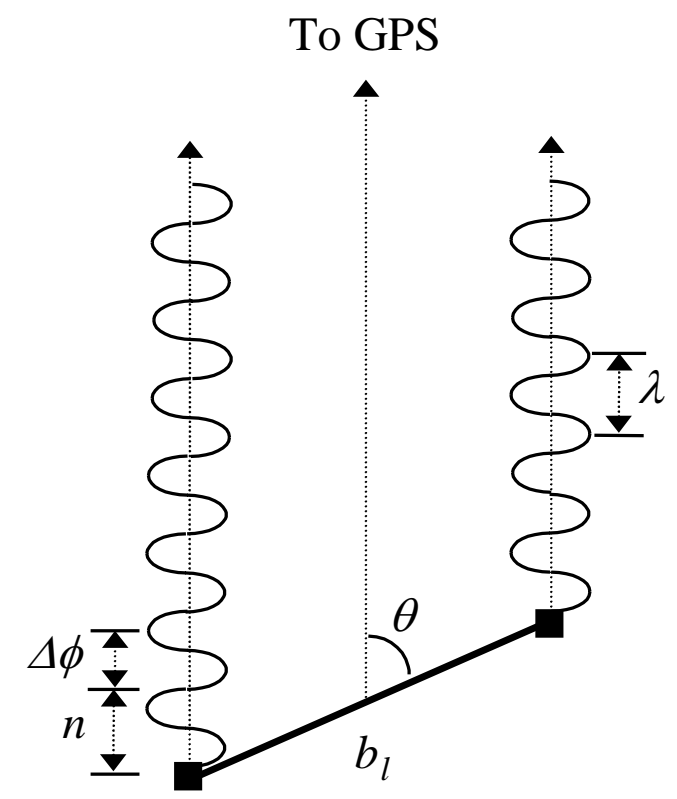

Figure 2: $\quad$ GPS Wavelength and Wavefront Angle 


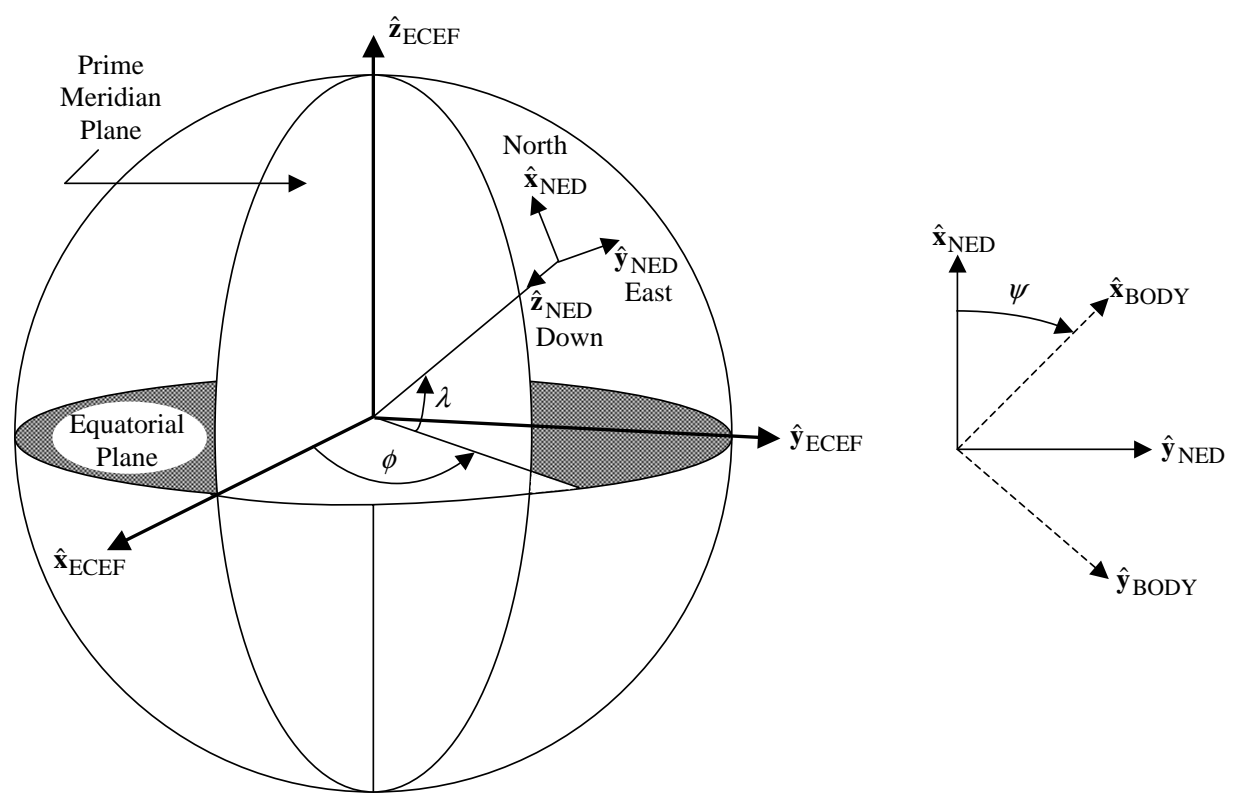

Figure 3: $\quad$ Earth-Centered-Earth-Fixed, North-East-Down and Body Frames 


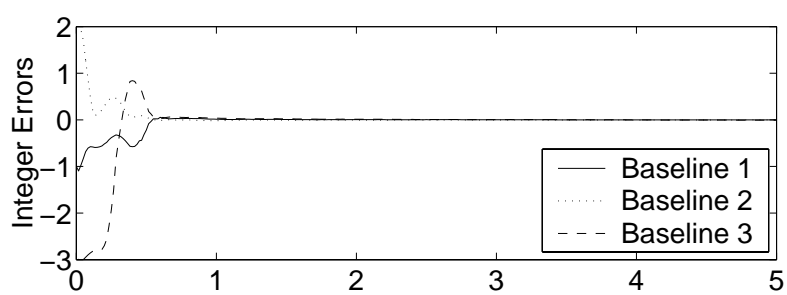

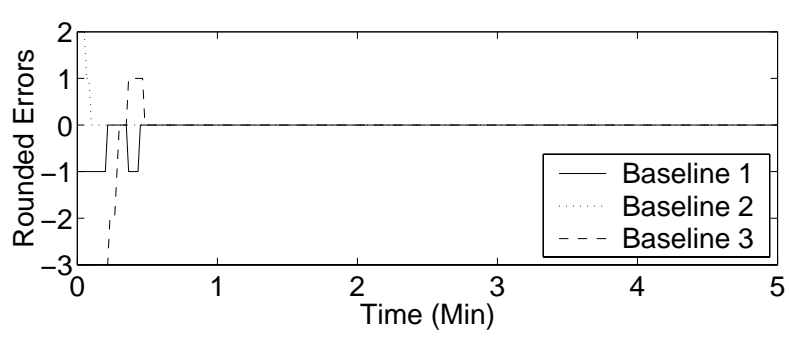

(i) Integer Errors

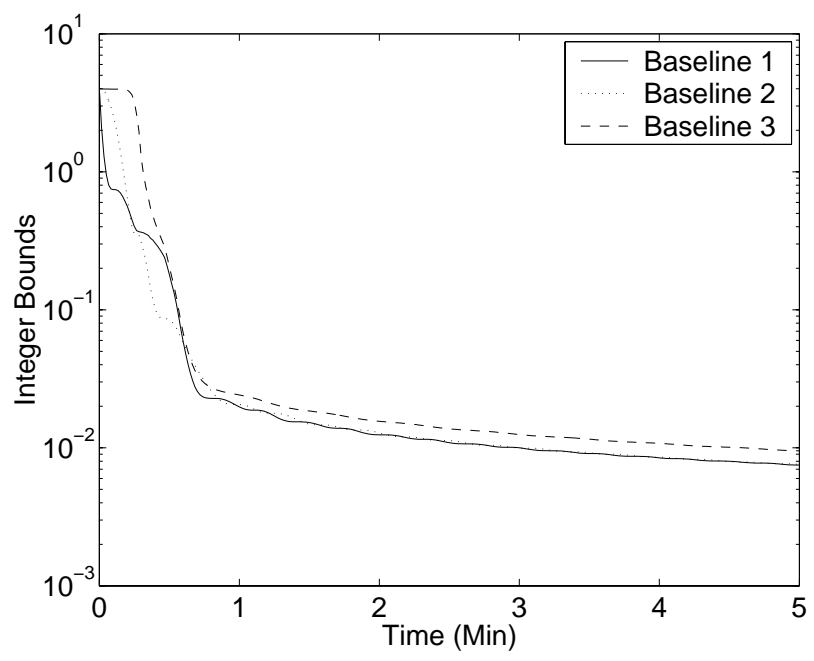

(ii) $3 \sigma$ Integer Bounds

Figure 4: $\quad$ Integer Errors and $3 \sigma$ Integer Bounds 

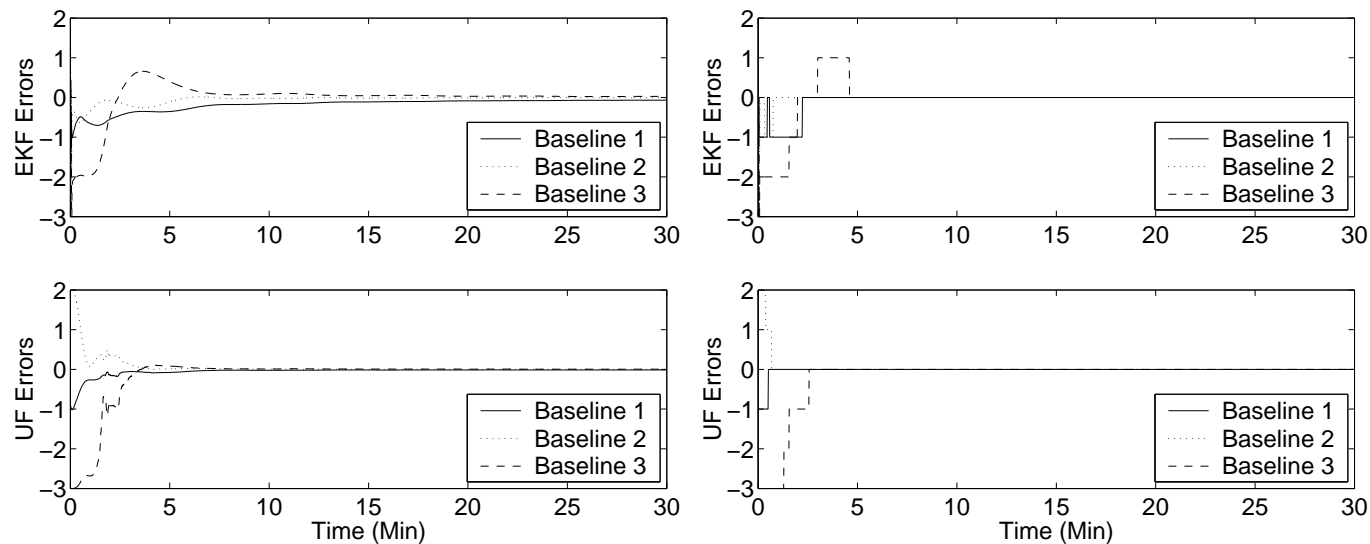

(i) Integer Errors

(ii) Rounded Integer Errors
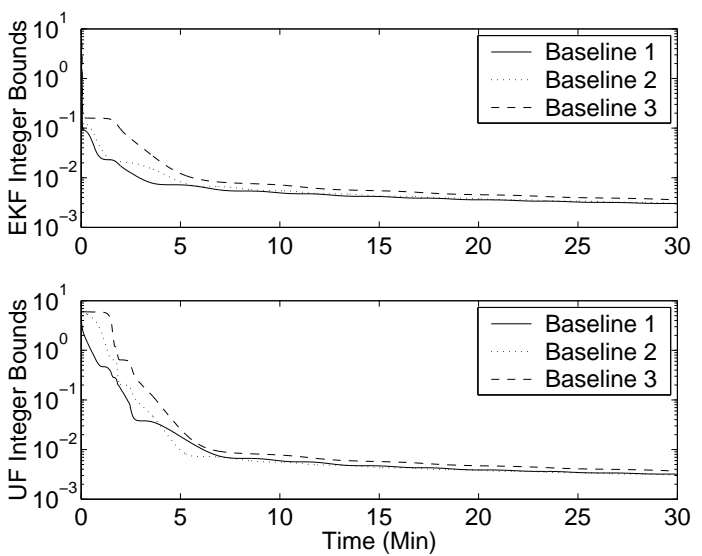

(iii) $3 \sigma$ Integer Bounds

Figure 5: $\quad$ EKF and UF Integer Errors and $3 \sigma$ Integer Bounds 

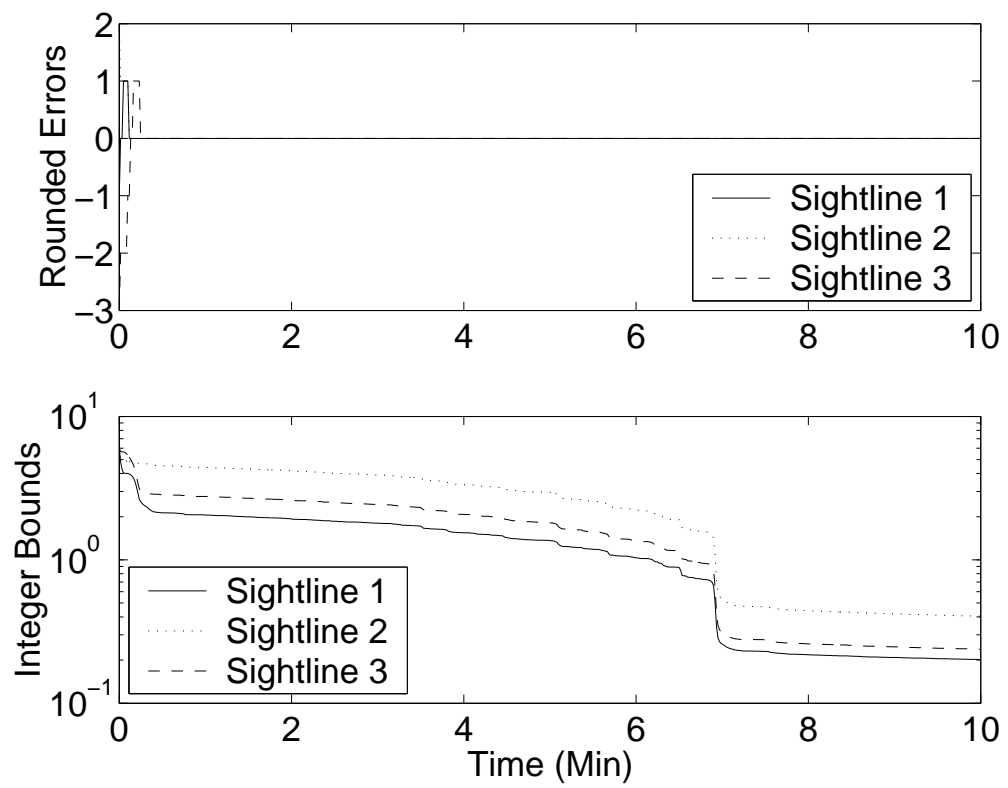

Figure 6: Rounded Integer Errors and $3 \sigma$ Integer Bounds for the UF 